\title{
Two new basal coelurosaurian theropod dinosaurs from the Lower Cretaceous Sao Khua Formation of Thailand
}

\author{
Adun Samathi, Phornphen Chanthasit, and P. Martin Sander
}

Acta Palaeontologica Polonica 64 (2), 2019: 239-260 doi:https://doi.org/10.4202/app.00540.2018

Megaraptora is a clade of mid to large-sized theropods that are long-snouted, large-clawed, highly pneumatized, and have long and gracile metatarsals. The basal member was reported from the Barremian of Japan. A more derived clade, the Megaraptoridae, is known from the Cenomanian to Santonian of Gondwana. Here two new basal coelurosaurs from the Lower Cretaceous Sao Khua Formation of Thailand are described and named as Phuwiangvenator yaemniyomi gen. et sp. nov. and Vayuraptor nongbualamphuensis gen. et sp. nov. Phuwiangvenator is a megaraptoran coelurosaur and diagnosed by the ventrally flat sacral vertebrae with sulci in the anterior and posterior region of the centra and the anterior rim of metatarsal IV sloping proximolaterally to distomedially and being much lower than that of metatarsal III anteriorly. Vayuraptor is a basal coelurosaur and diagnosed by its astragalus which has two horizontal grooves, two fossae at the base of the ascending process, the ascending process being straight laterally and straight and parallel medially with the medial rim sloping to the tip laterally, and a long and slender astragalar ascending process. Although the position of the basal coelurosaur Vayuraptor remains unclear and must await further discovery, megaraptoran affinities are likely. The Early Cretaceous megaraptoran fossil record has been recovered from the Barremian to Aptian of Asia. All Asian megaraptorans might be a monophyletic clade or a paraphyletic series relative to the Megaraptoridae. Several specimens have been reported from the Aptian to mid-Cretaceous of Australia, and one report from the Albian of South America. These fossils show a high diversity of the Early Cretaceous megaraptorans and a wide distribution during that time. The clade then became more provincial in the Late Cretaceous.

Key words: Dinosauria, Theropoda, Coelurosauria, Megaraptora, Phuwiangvenator, Vayuraptor, Cretaceous, Thailand.

Adun Samathi [asamathi@gmail.com] and P. Martin Sander [martin.sander@uni-bonn.de

], Division of Paleontology, Institute of Geo sciences, Rheinische Friedrich-Wilhelms Universität Bonn, Nussallee 8, Bonn, 53115, Germany. Phornphen Chanthasit [aom025@gmail.com], Sirindhorn Museum, Department of Mineral Resources, 200 Moo 11, Kalasin, 46140, Thailand. 
This is an open-access article distributed under the terms of the Creative Commons Attribution License (for details please see creativecommons.org), which permits unrestricted use, distribution, and reproduction in any medium, provided the original author and source are credited.

FoF Full text $(1,275.8 \mathrm{kB})$

FoF Supplementary file $(333.6 \mathrm{kB})$ 